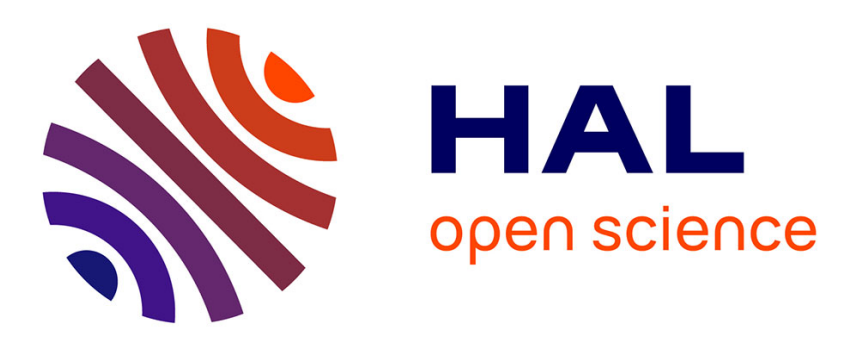

\title{
ORBITAL MOTION OF 3He-A IN THE PRESENCE OF A HEAT CURRENT
}

\author{
J. Hook
}

\section{To cite this version:}

J. Hook. ORBITAL MOTION OF 3He-A IN THE PRESENCE OF A HEAT CURRENT. Journal de Physique Colloques, 1978, 39 (C6), pp.C6-17-C6-18. 10.1051/jphyscol:1978608 . jpa-00217500

\section{HAL Id: jpa-00217500 https://hal.science/jpa-00217500}

Submitted on 1 Jan 1978

HAL is a multi-disciplinary open access archive for the deposit and dissemination of scientific research documents, whether they are published or not. The documents may come from teaching and research institutions in France or abroad, or from public or private research centers.
L'archive ouverte pluridisciplinaire HAL, est destinée au dépôt et à la diffusion de documents scientifiques de niveau recherche, publiés ou non, émanant des établissements d'enseignement et de recherche français ou étrangers, des laboratoires publics ou privés. 


\title{
ORBITAL MOTION OF ${ }^{3} \mathrm{He}_{\mathrm{A}}$ A IN THE PRESENCE OF A HEAT CURRENT
}

\author{
J.R. Hook \\ Physics Department, Manchester University, Manchester M13 9PL, U.K.
}

Rêsumé.- Nous avons résolu numériquement $l$ 'équation du mouvement du vecteur $\ell$ pour $1{ }^{3}$ He-A dans $1 e$ cas où le fluide est contenu entre deux plans parallèles infinis, entre lesquels circule un courant de chaleur. Des solutions périodiques dans le temps ont été obtenues, donnant une explication possible des mouvements orbitaux observés par Paulson, Krusius et. Wheatley $/ 1 /$.

Abstract. - We have solved numerically the equation of motion of the $\ell$ vector for ${ }^{3} \mathrm{He}-\mathrm{A}$ for the ćase where the fluid is contained between two infinite parallel planes a:d there is a flow of heat between the planes. Solutions periodic in time are obtained which provide a possible explanation of the persistent orbital motions observed by Paulson, Krusius and Wheatley $/ 1 /$.

Since the discovery of the superfluid phases of ${ }^{3} \mathrm{He}$, the interesting phenomena associated with the spin dynamics have been widely investigated. It has only recently been realized that the orbital dynamics of ${ }^{3} \mathrm{He}-\mathrm{A}$ is also a fruitful source of new phenomena; the decay of a supercurrent through motion of the $\underline{l}$ texture $/ 2 /$ and the persistent orbital motions observed in the sound attenuation measurements of Paulson, Krusius and theatley /1/ (referred to as PKW) are two examples of this. The orbital motions described in this paper provide a possible explanation of the experiments of PKW .

We have investigated solutions of the equation of motion of $\underline{\ell}$ when the fluid is contained between two infinite parallel planes (at $\mathrm{Z}=\mathrm{o}$ and $\mathrm{Z}=\mathrm{d}$ ) and there is counterflow $\underline{w}=\underline{v}_{n}-\underline{v}_{s}$ between the planes such as would be produced by a heat current. We assume dipole-locking (valid for $d>6 \mu \mathrm{m}$ ) and spatially uniform $\underline{v}_{n}$, in which case the equation of motion of $\ell$ in the Ginzburg-Landau region becomes

$$
\begin{aligned}
& \frac{2 \cdot \mu}{\rho_{s_{11}}}\left(\frac{\partial \underline{l}}{\partial t}+\left(\underline{v}_{n} \cdot \underline{\nabla}\right) \underline{\ell}\right)=-5 \underline{\ell} \times\left(\underline{\ell} \times \nabla^{2} \underline{\ell}\right)- \\
& -4 \underline{\ell} \times(\underline{W} \cdot \underline{\nabla}) \underline{\ell} \\
& +2 \underline{\ell} \times(\underline{\ell} \times \operatorname{cur} 1 \underline{\ell})(\underline{\ell} \cdot \operatorname{cur} 1 \underline{\ell}+\underline{\ell} \cdot \mathrm{W}) \\
& +2 \underline{\ell} \times(\underline{\ell} \times \underline{W})(\underline{\ell} . \operatorname{cur} 1 \underline{\ell}-\underline{\ell} . \underline{W}) \\
& +2 \underline{\ell} \times \underline{\nabla}(\underline{\ell} \cdot \underline{W})
\end{aligned}
$$

where $\mu$ is the orbital viscosity and we have put $H / 2 m=1$. This equation differs form that derived by us in a previous publication /3/ (referred to as
I) by the inclusion of terms, erroneously omitted in $I$, arising from the $\ell$ dependence of the tensors $\mathbb{T}^{\mathrm{T}}$ and $\mathrm{T}^{\mathrm{n}}$ introduced in $\mathrm{I}$. The corrected equation agrees with that given by other authors, e.g. Hu and Saslow $/ 4 /$.

We assume that $\underline{\ell}$ and $\underline{W}$ vary only in the $z$ direction; conservation of matter then determines the $Z$ variation of $W$ for a given $\ell$ and also implies that $v_{s} \gg v_{n}$ in the Ginzburg-Landau region. The solutions described below were all obtained for $\underline{v}_{n}=o$ but inclusion of a small $\underline{v}_{n}$ does not cause any significant change. It is convenient to describe the solutions using the polar angles $\theta(z, t)$ and $\phi(2, t)$ of $\ell$ with respect to $z$. We use the dimensionless units $t^{*}=\rho_{s} w_{0}^{2} t / 2 \mu$ and $z^{*}=W_{o} Z(2 m / K)$, where $W_{0}$ is the value of $|\underline{W}|$ at $z=0$, $d$. We consider both possible types of boundary conditions :

a) $\theta=0$ at $z=0, d$ ( 0 - o solutions);

b) $\theta=0$ at $z=0$ and $\theta=\pi$ at $z=d(0-\pi$ solutions).

The solutions described briefly below were obtained by numerical integration of equation (1) by methods which will be described in a forthcoming publication.

a) $0-\pi$ domain wall solutions.- We have found precessing domain wall solutions of equation (1) similar to those discussed in $I$ with $\theta=\theta(Z)$ i.e. independent of time.

$$
\phi=\phi(Z)+\omega^{*} t
$$

The precessional frequency $\omega^{\star}$ of these solutions is shown as a function of $1 / d^{*}$ in figure 1 . It can be seen that $\omega^{\star} \propto 1 / d^{*}$ for small $d^{*}\left(\omega \propto h_{0} \rho_{s} W_{o} / m \mu d\right.$ in usual units) but $\omega^{\star}$ tends to a constant value for large $d^{*}$ ( $\omega \propto \rho_{s \| l} W_{0}{ }^{2} / \mu$ in usual units). For 
For small $\mathrm{d}^{\mathbf{t}}$ the domain wall is in the centre of the channel whereas for $d^{\star} \gtrsim 17$ the domain wall moves away from the middle.

b) o - o solutions.- In contrast to the situation for the incorrect equations derived in $I$, the $\theta=$ o everywhere solution of equation (1) appears to be stable against small perturbations for all $\mathrm{d}^{*}$. It is likely to become unstable in wide channels at lower temperatures and can also be rendered unstable by the application of a small magnetic field in the $\mathrm{z}$ direction. We have investigated this latter possibility and will describe some of the exotic textures which result in a future publication. For $d^{\star} \gtrsim 21$ equation (1) has, in addition to the $\theta=0$ solution, a more interesting time dependent - - o solution which looks approximately like a o - $\pi$ domain wall followed by a $\pi$ - o domain wall, although in this case $\theta$ is in fact slightly time dependent. The two domain walls precess in opposite senses and the precession frequency which is shown in figure 1 , is approximately independent of $d^{\star}$ for $d^{*} \gtrsim 24$ (corresponding to $\omega \propto \rho_{s}, w_{0} / \mu$ in usual units) and close to the frequency of precession of a single $0-\pi$ domain wall in a wide channel.

The o- $\pi$ domain wall and the 0 - 0 time dependent solution would cause periodic changes in sound attenuation similar to those seen by PKW. To demonstrate this we plot on figure 2 for a typical o - $\pi$ domain wall the spatial average of $l_{x}^{2}$ over the channel as a function of time, which should reflect changes in the attenuation for sound waves travelling in the $x$ direction. The oo time dependent solution is however more constitent with the experimental results of PKW insofar as

a) The rotating magnetic fields needed to turn on the motion would correspond to the need to rotate $\ell$ through a large angle to set up a o - $\pi$ - o texture.

b) $\omega \propto \rho_{s} W_{0}^{2} f \mu \rightarrow \omega \propto\left(1-T / T_{c}\right)^{-5 / 2}$, close to the dependence observed by PKW. The order of magnitude of the frequency is also correct although detailed comparison is impossible because of the complicated geometry of the experiments.

c) The disappearance of the motion on cooling would correspond to the dimensionless thickness, which varies as $\left(1-T / T_{c}\right)^{-1}$, decreasing through the value below which the oscillations do not occur.

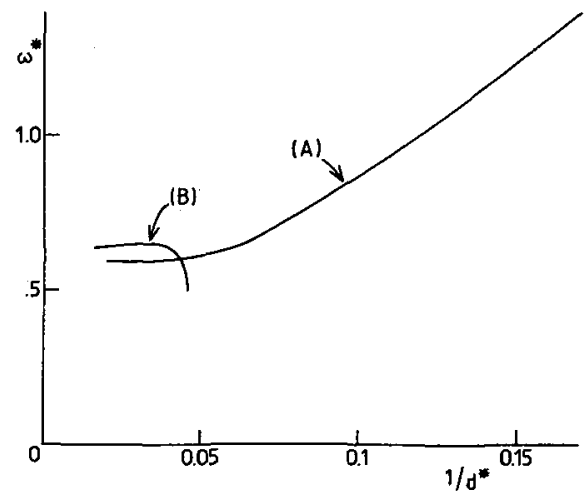

Fig. 1 : Precession frequency $\omega^{*}$ for $(A) \circ-\pi$ domain walls and $(B) 0^{\circ}-0$ time dependent solutions as a function of $1 / \mathrm{d}^{*}$.

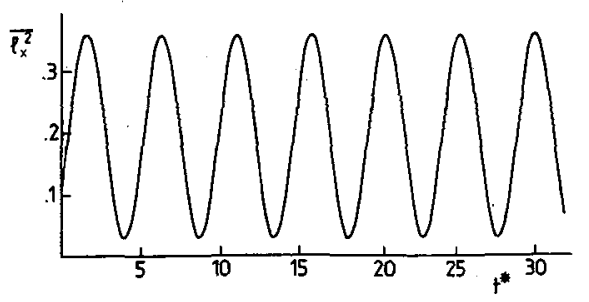

Fig. 2 : The average value of $\ell^{2}$ within the channel as a function of $t^{t}$ for a $o^{x}-\pi$ domain wall in a channel with $d^{*}=15$.

\section{References}

/1/ Paulson, D.N., Krusius, M., and Wheatley, J.C., Phys. Rev. Lett. 37 (1976) 803.

/2/ Mermin, N.D., Quantum Fluids and Solids ed. by Trickey S.B., Adams, E.D., and Duffy, J.w. (Plenum 1977) p. 3.

/3/ Hall, H.E. and Hook, J.R., J. Phys. C 10 (1977) L91.

14/ Hu, C.R. and Saslow, W.M., Phys. Rev. Letts. 38 (1977) 605 . 